\title{
EVALUACIÓN DEL DESARROLLO DE LAS COMPETENCIAS CIENTÍFICAS EXPLICAR E INDAGAR EN LA APLICACIÓN DE UN TRABAJO PRÁCTICO SOBRE FOTOSÍNTESIS
}

\section{Evaluation of developing scientific competences explain and Inquiry in the application of a practical work about Photosynthesis}

Recibido: 01-04-2010
Aceptado: 22- 06-2010

Por: *Sonia Esmeralda Garnica Mojica, ${ }^{* *}$ Judith Arteta de Molina ${ }^{1}$

Resumen: Esta investigación tuvo como objetivo principal evaluar el desarrollo de competencias científicas, específicamente indagar y explicar en la aplicación de un trabajo práctico sobre fotosíntesis en el marco de la enseñanza para la comprensión.

La Metodología utilizada para esta investigación tenía un carácter cualitativo, orientada básicamente a la práctica educativa: “Decisión y cambio" cumple una función instrumental, por cuanto tiene una aplicación directa en el cambio y mejora de la práctica lo que se conoce como investigación acción.

En este trabajo se evaluaron las competencias a través de una guía de laboratorio lo que permitió verificar que el procedimiento permitía el desarrollo de algunos de los indicadores de desempeño de las competencias y para dar alcance a todos ellos fue necesario la reevaluación y mejoramiento de la guía por medio de cuestionamientos orientados hacia la formulación de preguntas a partir de los fenómenos observados.

Palabras claves: Competencias, Indagar, Explicar, trabajo práctico, fotosíntesis, enseñanza para la comprensión.

Abstract: The main objective of this research was to evaluate the development of scientific competences, specifically to inquiry and explain in the application of a practical work on photosynthesis in the context of teaching for understanding.

The used methodology for this research was qualitative, focused mainly on the educational practicum: "Decision and Change" plays an instrumental role as it has direct application in the change and improvement of the practicum what is known as action research. 
This paper evaluates skills through a laboratory guide which allowed to check that the procedure made possible the development of some competence performance indicators. To reach out to all of them it was necessary the re-assessment and improvement of the guide through targeted questions towards questions formulation based on the observed phenomena.

Key words: Competences, Inquiry, Explain, Practical work, photosynthesis, teaching for understanding.

1 *Licenciada en Biología Universidad de La Salle. soniagarnicam@hotmail.com

** Profesora de Biología. Universidad Pedagógica Nacional. juditharte@yahoo.com

\section{INTRODUCCIÓN}

El cotidiano quehacer del docente lo lleva a cuestionarse acerca de la manera en que se puede aportar para hacer caso a las demandas de la sociedad y crear conciencia a los estudiantes de su posición crítica antes las situaciones que ella les impele. Esta constante reflexión posibilita comprender lo que hacemos en el aula e implementar nuevas propuestas o iniciar investigaciones que mejoren la educación en las instituciones educativas; de otra forma no se hará nunca la tan mencionada educación con calidad y con ánimos emprenderistas. ¿Pero qué podemos hacer los docentes desde el aula de clase, junto con las políticas de las instituciones y del país, para contribuir en la formación de personas altamente competitivas y productivas?

En el aula de clase es común encontrar estudiantes que memorizan los conceptos sin entender qué significan ni cómo se relacionan y profesores que a través de la transmisión de conceptos llevan a cabo su práctica. Las situaciones diarias demuestran que es necesario cambiar este tipo de educación para que nuestros estudiantes se apropien del conocimiento para la transformación de su realidad descubriendo la relación entre lo que aprenden y su cotidianidad.

En el caso específico de los conocimientos del área de las Ciencias Naturales, y en especial de la Biología es importante, comprender los conceptos y aún más, es imperiosa la necesidad de ejecutar procesos de investigación y aplicación a través de trabajos prácticos para el desarrollo de las competencias científicas, actitudes para explorar fenómenos y para resolver problemas.

Según Blythe (1999) el modelo de enseñanza para la comprensión, se convierte en un marco orientador para que el estudiante demuestre qué entiende de un tópico específico y sea capaz de ampliarlo de una forma innovadora. Esta estrategia se basa en cuatro elementos: Tópicos Generativos, Metas de Comprensión, Desempeños de Comprensión y Evaluación Diagnóstica. Dentro de los desempeños de compresión se encuentran una serie de actividades que pueden facilitar el proceso de aprendizaje, ejemplo de ellos, se pueden 
resatar las prácticas de laboratorio, las cuales han mostrado en desde su ejecución buenos resultados al mostrar a través de montajes sencillos procesos complejos de entender.

Los trabajos prácticos para la enseñanza y el aprendizaje de las ciencias son ayuda inestimable para la comprensión de los planteamientos teóricos de la ciencia y el desarrollo del razonamiento científico por parte de los alumnos, facilitan la comprensión del conocimiento y su significado, son insustituibles para la enseñanza y el aprendizaje de procedimientos científicos, pueden ser una base sólida para desarrollar algunas actitudes fundamentales relacionadas con el conocimiento científico (Del Carmen. 2000). Se constituyen como una oportunidad para el trabajo en equipo y el desarrollo de las actitudes y la aplicación de normas propias del trabajo experimental: planificación, orden, limpieza, seguridad, etc (Caamaño, 2003).

En ciencias los estándares básicos definen competencias que tienen un énfasis en el desarrollo de las habilidades y actitudes científicas por parte de los estudiantes. Los trabajos prácticos facilitan que el estudiante sea capaz de reconocer y diferenciar explicaciones científicas y no científicas acerca del funcionamiento del mundo y de los acontecimientos que en éste suceden, esto es, la capacidad crítica, presentar argumentos ante los hechos naturales y del diario vivir, establecer relaciones por medio de la creatividad. Estos estándares han definido que lo anteriormente descrito son solo algunos de los objetivos de la educación en Ciencias.

Para lograrlo de acuerdo con el ICFES y la Fundamentación en Ciencias Naturales (2007) se deben desarrollar en los estudiantes las competencias de Identificar, Indagar y Explicar, Comunicar, Trabajar en equipo, Disposición para reconocer la dimensión social del conocimiento y Disposición para aceptar la naturaleza cambiante del conocimiento.

Las competencias Explicar e Indagar dan cuenta de una forma particular del conocimiento Biológico por ser una forma de realización específica de la comprensión de los fenómenos y del quehacer en el área, el desarrollo de estas competencias permite que el estudiante vaya avanzando paulatinamente en el conocimiento del mundo desde una óptica que depende de la posibilidad de dudar, de preguntarse acerca de lo que se observa para interactuar de manera lógica y propositiva en el mundo en que se desarrolla.

La búsqueda de explicaciones constituye una parte fundamental de la actividad del ser humano y puede considerarse como inherente al deseo de entender el mundo, éstas se construyen dentro del marco de sistemas como conceptos, que han sido propuestos y acogidos por la comunidad científica.

En la formulación de preguntas se establece la capacidad de aprender a aprender. Una vez se logra formular una pregunta relativamente precisa, se puede proceder a establecer un método de trabajo para resolverla (Fundamentación en Ciencias Naturales, 2007). 
En cuanto a la fotosíntesis estudios recientes (Charrier, 2006) han permitido poner en evidencia la existencia de graves problemas de enseñanza y aprendizaje, y la génesis de las mismas, han despertado un gran interés entre los investigadores.

La presente investigación pretendió, desde una práctica sencilla de laboratorio acerca de la fotosíntesis que se ha venido realizando desde hace dos años con los estudiantes del grado noveno del Colegio Colombo Gales en el marco de la enseñanza para la comprensión , evaluar el desarrollo de competencias científicas, explicar e indagar y determinar si realmente se están alcanzando los objetivos propuestos, tanto en la guía de laboratorio como en la planeación que se realiza al iniciar el año escolar.

Por medio de este escrito se pretende mostrar los resultados obtenidos durante la evaluación del desarrollo de las competencias científicas, indagar y explicar, en tres momentos fundamentales. El análisis de la guía de laboratorio, su desarrollo y, por último, la entrega del reporte del laboratorio por parte de los estudiantes.

\section{MATERIALES Y MÉTODOS}

En el siguiente apartado se describirá el proceso llevado a cabo durante la cualificación, análisis, revisión y aplicación del trabajo práctico sobre fotosíntesis, centrado en la descripción y los hallazgos de fenómenos observados (Cerda, 2005), este proceso se hizo en tres fases : Diseño, Ejecución e interpretación de resultados

\section{Definición del tipo de Investigación}

De acuerdo con el problema en estudio, esta investigación es de tipo cualitativo. El instrumento y técnica para la recolección de datos fue el análisis de documentos que incluye: guía de laboratorio y posteriormente una filmación, observación del desarrollo de las prácticas y trabajos o reportes de laboratorios presentados por los estudiantes.

\section{Generalidades y Contexto de la Investigación}

Se determinaron algunos aspectos importantes que pueden influir en el desarrollo de esta investigación, entre estos aparecen la edad de los estudiantes, el colegio en donde se desarrolló la investigación. Se trabajó con estudiantes de tres Grados de Noveno A, B y C en promedio de 25 a 30 estudiantes en cada salón para un total de 83, cuyas edades oscilan entre los 13 a 16 años del Colegio Colombo Gales (calendario B), ubicado al Costado Occidental del Aeropuerto Guaymaral.

Se escogió la guía de laboratorio titulada "Photo finish", tomada del Libro Biology. Exploring Life (Campbell, 2004) utilizado como guía de estudio para el grado noveno ya que es una de las prácticas que se programó para el último periodo académico, para el 
desarrollo de la práctica de laboratorio los estudiantes se agruparon en grupos de seis estudiantes y la grabación se realizo con los tres grupos ésta práctica se desarrollo en el primer semestre del año 2008, y que coincidió con la metodología planteada y se pudo realizar sin afectar el plan de estudios del Colegio y la programación bimestral que se realiza con anticipación.

El análisis de esta práctica de laboratorio se presenta como una opción oportuna para evaluar y reestructurar las estrategias aplicadas en el aula de clase para el grado noveno y dar alcance a las competencias propuestas por medio de un trabajo práctico para el área de ciencias naturales del Colegio Colombo Gales. Este puede ser un aporte significativo para los maestros en ejercicio para hacer que se cuestionen y replanteen las actividades que desarrollan en el aula con sus estudiantes.

\section{Revisión Teórica}

En la revisión teórica se realizó una sistematización de los referentes teóricos acerca de las posibles categorías que se podrían plantear para el apropiado análisis de la guía de laboratorio. Se identificaron elementos involucrados con la enseñanza y con el aprendizaje de los conceptos, procedimientos y actitudes. También marcos de referencia en cuanto a la comprensión, los trabajos prácticos, las competencias y el proceso de fotosíntesis.

\section{EJECUCIÓN}

\section{Fases iniciales de ejecución}

\section{Escogencia de la práctica de laboratorio.}

Para la escogencia de la práctica de laboratorio a analizar se tuvo en cuenta que estuviera programada para el primer semestre de 2008 y que el tema ofreciera dificultades de comprensión. (Ver Anexo 1)

Esta guía se ha aplicado en años anteriores a los alumnos del grado noveno dentro de la Unidad de Fotosíntesis por lo cual fue importante analizar los objetivos y evaluar si la guía permite el logro de ellos y de las habilidades investigativas propuestas.

Los procesos de fotosíntesis y respiración celular hacen parte de los Lineamientos curriculares para el área de Ciencias Naturales, en el Colegio, por lo que se han abordado en el aula de clase desde diferentes temáticas y en diferentes niveles desde preescolar. Aún así, es común encontrar estudiantes que tienen falencias en la aplicación, se hace evidente la problemática y aún son numerosas las cuestiones a dilucidar en torno a la enseñanza y aprendizaje de la fotosíntesis y respiración celular. En el área de Ciencias para los grados 9 
a 12, los siguientes temas son considerados conocimientos fundamentales: la naturaleza química de los procesos biológicos; materia y energía en los sistemas biológicos: la base molecular de la herencia y la variación, diversidad y evolución de las especies. (Stone, 1998:53)

\section{Análisis Cualitativo de la guía de laboratorio}

Inicialmente a partir del marco teórico se obtuvieron los indicadores tanto de las competencias explicar e indagar como los de la comprensión y su respectiva codificación para contrastarlos con la guía de laboratorio y determinar si se favorecía o no de cada indicador en la guía.

Luego se tuvieron en cuenta los contenidos teórico-conceptual de la guía de laboratorio, los contenidos procedimentales y los contenidos actitudinales del trabajo práctico teniendo en cuenta las habilidades para evaluar las actividades de laboratorio según del Carmen (2000)

Para un acercamiento epistemológico se realizó una síntesis de algunos aspectos epistemológicos acorde con el análisis planteado por Canguilhem (1976).

De acuerdo con los referentes teóricos presentados por Del Carmen (2000) y Caamaño (2003) se realizó una clasificación y evaluación en cuanto a las dimensiones y objetivos perseguidos que se pretenden desarrollar con la práctica de laboratorio.

\section{Valoración Inicial}

Con base en el análisis cualitativo, se planteó una serie de observaciones iniciales referentes a sus debilidades, limitantes y fortalezas en aras de generar una nueva propuesta didáctica que cumpliera con los desempeños propuestos desde el marco teórico.

\section{Fases finales de Ejecución}

\section{Aplicación de la práctica de laboratorio}

De acuerdo con las fechas establecidas se llevó a cabo la práctica de laboratorio con los tres cursos de grado noveno.

\section{Filmación}

Durante las prácticas de laboratorio los estudiantes se agruparon de a seis o cinco y se realizó la filmación de cada curso en el salón del laboratorio. 


\section{Recolección de Información y análisis}

- Durante la práctica de laboratorio

Se elaboraron algunas pautas para observar los desempeños en cuanto a las competencias explicar e indagar y las dimensiones de comprensión mientras los estudiantes estaban realizando el trabajo práctico, los resultados fueron registrados en las tablas diseñadas para la recolección de la información posteriormente con la filmación se comparó la información y se completaron las tablas.

- Después de la práctica de laboratorio

Se utilizaron los mismos criterios para valorar los registros de la práctica en los reportes de laboratorio presentados por los estudiantes, se tomo como muestra 15 reportes (cinco de cada curso) se numeraron ascendentemente y se valoró cada una de las unidades de análisis que requería respuesta por parte de los estudiantes para esto se contrastó con los referentes teóricos el nivel de comprensión, el desarrollo de las competencias y el manejo conceptual.

\section{- $\quad$ Triangulación}

Se realizó una triangulación de los datos obtenidos: Filmación, observación y reportes de laboratorio y se logró contrastar toda la información para llegar a unas generalizaciones finales.

\section{Interpretación de los resultados}

A partir de los resultados obtenidos tanto con la guía de laboratorio, durante el trabajo práctico y posteriormente con los reportes de laboratorio se realizo un análisis e interpretación teniendo en cuenta los referentes teóricos.

\section{RESULTADOS Y DISCUSIÓN}

Los resultados que se mostrarán a continuación recogen los diferentes hallazgos producidos en los distintos momentos del proceso de investigación.

Fases iniciales de ejecución

Análisis Cualitativo de la guía de laboratorio

Competencias científicas de Explicar e Indagar en el trabajo práctico. 
Los Indicadores para las competencias científicas explicar e indagar se tomaron textualmente del planteamiento hecho en la Fundamentación Conceptual para el área de Ciencias Naturales (ICFES, 2007). Para la Competencia Explicar se utilizó el número 1 y para sus indicadores letras organizadas alfabéticamente, para la competencia Indagar el número 2 y sus indicadores letras organizadas alfabéticamente.

Los Indicadores para las competencias científicas explicar e indagar

\begin{tabular}{|c|c|}
\hline 1. EXPLICAR & INDICADORES \\
\hline \multirow{6}{*}{$\begin{array}{l}\text { Según ICFES (2007) la } \\
\text { fundamentación conceptual del } \\
\text { área de ciencias naturales plantea } \\
\text { que: explicar es la capacidad para } \\
\text { construir y comprender } \\
\text { argumentos, representaciones o } \\
\text { modelos que den razón de } \\
\text { fenómenos. }\end{array}$} & a. Dar razón de fenómenos o problemas. \\
\hline & b. Búsqueda de explicaciones. \\
\hline & c. formulación de preguntas. \\
\hline & d. producción de razones. \\
\hline & e. causa y relación con fenómenos. \\
\hline & f. Fomentar la actitud crítica. \\
\hline 2. INDAGAR & INDICADORES \\
\hline \multirow{15}{*}{$\begin{array}{l}\text { Según la fundamentación } \\
\text { conceptual del área de ciencias } \\
\text { naturales planteada por el ICFES } \\
\text { Indagar es la capacidad para } \\
\text { plantear preguntas } \\
\text { procedimientos adecuados y para } \\
\text { buscar, seleccionar, organizar e } \\
\text { interpretar información relevante } \\
\text { para dar respuesta a esas } \\
\text { preguntas. }\end{array}$} & a. observar detenidamente la situación \\
\hline & b. Establecer preguntas pertinentes \\
\hline & c. Establecer elementos para comprenderla \\
\hline & d. Búsqueda de información. \\
\hline & e. Planear un experimento sencillo \\
\hline & f. Planteamiento de nuevas preguntas \\
\hline & g. Buscar relaciones de causa-efecto \\
\hline & h. Hacer predicciones \\
\hline & $\begin{array}{l}\text { i. Realizar mediciones y organizar y analizar } \\
\text { resultados. }\end{array}$ \\
\hline & $\begin{array}{l}\text { j. La capacidad de buscar, recoger, seleccionar } \\
\text { información }\end{array}$ \\
\hline & k. Consulta de libros \\
\hline & 1. Predecir \\
\hline & m. identificar variables \\
\hline & n. Seleccionar experimentos adecuados \\
\hline & o. Analizar resultados \\
\hline
\end{tabular}

Tabla 1. Indicadores de las competencias Explicar e indagar para el análisis de la práctica.

Ejemplo de codificación usada:

\begin{tabular}{|c|l|}
\hline CODIFICACIÓN & \multicolumn{1}{|c|}{ INTERPRETACIÓN } \\
\hline $1-b$ & $\begin{array}{l}\text { Competencia: 1- Explicar } \\
\text { Indicador: b-Búsqueda de explicaciones }\end{array}$ \\
\hline $2-b$ & Competencia: 2-Indagar \\
\hline
\end{tabular}




\begin{tabular}{|l|l|}
\hline & $\begin{array}{l}\text { Indicador: b-Establecer preguntas } \\
\text { pertinentes }\end{array}$ \\
\hline
\end{tabular}

Tabla 2. Ejemplo de Utilización de los Indicadores Explicar e Indagar

Se determinaron las dimensiones de comprensión (Tabla 2) para evaluar el trabajo práctico, las competencias científicas de explicar e indagar en el trabajo práctico (Tabla 3). Para cada uno se utilizaron números y letras organizadas alfabéticamente.

Dimensiones de Comprensión para la guía analizada

\begin{tabular}{|l|}
\hline \multicolumn{1}{|c|}{ DIMENSIONES DE LA COMPRENSIÓN } \\
\hline \multicolumn{1}{|c|}{ 1. CONOCIMIENTO } \\
\hline a. Manejo de conceptos disciplinarios \\
\hline b. Redes altamente organizadas \\
\hline c. Dominio del tema \\
\hline \multicolumn{2}{|c|}{ 3. PROPÓSITO } \\
\hline a. Discusión \\
\hline b. Lo hace por el mismo \\
\hline c. Preguntas al professor \\
\hline d. Preguntas a los compañeros \\
\hline \multicolumn{2}{|c|}{ 4. FORMA } \\
\hline a. Reflexión \\
\hline b. Se desenvuelve fácilmente \\
\hline \\
\hline a. Toma en cuenta la opinión de los demás \\
\hline
\end{tabular}

Tabla 3. Elementos de la enseñanza de la comprensión para el análisis de la práctica.

Teniendo en cuenta lo anterior, se verificaron los Indicadores para las competencias explicar e Indagar e indicadores para las dimensiones de comprensión en la guía de laboratorio (para estos últimos solo se utilizó codificación), según se muestra en la tabla 4. 
Unidades de análisis establecidas para la guía de laboratorio

\begin{tabular}{|c|c|c|}
\hline GUÍA DE LABORATORIO. & $\begin{array}{l}\text { INDICADORES } \\
\text { PARA LAS } \\
\text { COMPETENCIAS } \\
\text { INDAGAR Y } \\
\text { EXPLICAR }\end{array}$ & $\begin{array}{l}\text { INDICADORES PARA } \\
\text { LAS DIMENSIONES DE } \\
\text { COMPRENSIÓN }\end{array}$ \\
\hline $\begin{array}{l}\text { 1. ¿Cuál hoja una joven o una } \\
\text { adulta fotosintetizará a una } \\
\text { tasa mas rápida? }\end{array}$ & 1-A Dar razón & $1-\mathrm{a}, 1-\mathrm{c}, 2-\mathrm{a}$ \\
\hline 2. Apreciación Global. & $\begin{array}{l}\text { 1-b Busqueda de } \\
\text { explicaciones } \\
\text { 2-i Realizar } \\
\text { mediciones } \\
\end{array}$ & $1-a, 1-c, 2-b, 3-a, 3-b$ \\
\hline 3. Introducción. & 2-k Consulta de libros & $2-b, 3-b$ \\
\hline 4. Actividad de Previa. & $\begin{array}{l}\text { 1-b Busqueda de } \\
\text { explicaciones } \\
\text { 2-a Observar } \\
\text { 2-j La capacidad de } \\
\text { buscar }\end{array}$ & 1-a,1-c,2-b,2-c,2-d,3-a,3-b \\
\hline 5. Una carrera de fotosíntesis & $\begin{array}{l}\text { 2-c Establecer } \\
\text { elementos para } \\
\text { comprenderla }\end{array}$ & $1-a, 1-b$ \\
\hline $\begin{array}{l}\text { 6. Ganando la carrera de la } \\
\text { fotosíntesis }\end{array}$ & $\begin{array}{l}\text { 2-c Establecer } \\
\text { elementos para } \\
\text { comprenderla }\end{array}$ & $1-a, 1-b$ \\
\hline $\begin{array}{l}\text { 7. a. ¿Si usted pudiera diseñar una } \\
\text { hoja que fotosintetice } \\
\text { "Velozmente", } \\
\text { características qué } \\
\text { (Piense acerca de la } \\
\text { estructura de una hoja y la } \\
\text { célula de la planta.) } \\
\text { Explique su razonamiento } \\
\end{array}$ & $\begin{array}{l}\text { 2-L Predecir } \\
\text { 1-b Busqueda de } \\
\text { explicaciones }\end{array}$ & $1-a, 1-c, 2-b, 3-a$ \\
\hline $\begin{array}{l}\text { 7. b. ¿Qué hoja de hiedra } \\
\text { fotosintetizará más rápido y } \\
\text { ganará la carrera? Explique } \\
\text { el por qué de su predicción. } \\
\text { (Ésta es su hipótesis.) }\end{array}$ & $\begin{array}{l}\text { 1-b Busqueda de } \\
\text { explicaciones } \\
\text { 2-1 Predecir } \\
\text { 2-g Relaciones de } \\
\text { causa-efecto } \\
\text { 2-h Hacer predicciones }\end{array}$ & $1 a, 1 c, 2 a, 2 b, 3 a$ \\
\hline $\begin{array}{l}\text { 7. c. Una tasa mide cómo una } \\
\text { cantidad cambia con el } \\
\text { tiempo. Dé un ejemplo de }\end{array}$ & $\begin{array}{l}\text { 1-a Dar razón } \\
\text { 1-e Causa y relación } \\
\text { 2-d Búsqueda de } \\
\end{array}$ & 1-a,1-c,2-a,2-b,2-c, 3-a,3-b \\
\hline
\end{tabular}




\begin{tabular}{|c|c|c|}
\hline $\begin{array}{l}\text { algo a lo que usted podría } \\
\text { medirle una proporción. } \\
\text { ¿Qué unidades de medida } \\
\text { serían (por ejemplo, } \\
\text { metros/segundos)? }\end{array}$ & información & \\
\hline 8. Materiales & $\begin{array}{l}\text { 2-c Establecer } \\
\text { elementos para } \\
\text { comprenderla }\end{array}$ & $1-a, 1-b$ \\
\hline $\begin{array}{l}\text { 9. } \\
\text { Procedimiento } \\
\text { Tiempo de salida de carrera: } \\
\text { Tabla de datos } 1 .\end{array}$ & $\begin{array}{l}\text { 2-a Observar } \\
\text { detenidamente la } \\
\text { situación } \\
\text { 2-i Realizar mediciones }\end{array}$ & $2-b, 3-b$ \\
\hline 10. Resultados de la Carrera: & 2-i Realizar mediciones & $2 b, 3 b$ \\
\hline $\begin{array}{l}\text { 11. Análisis y Conclusiones: } \\
\text { Antes de que la carrera } \\
\text { empezara.....Ajustando sus } \\
\text { hipótesis basándose en los } \\
\text { datos. }\end{array}$ & $\begin{array}{l}\text { Método Hipotético- } \\
\text { deductivo }\end{array}$ & \\
\hline 11. a. ¿Predijo usted al ganador? & $\begin{array}{l}\text { 1-f Fomentar actitud } \\
\text { crítica } \\
\text { 1-a Dar razón }\end{array}$ & 1-a,1-c,2-a,3-a,4-a \\
\hline $\begin{array}{l}\text { 11. b. Calcule la tasa de } \\
\text { fotosíntesis del ganador } \\
\text { completando el cálculo } \\
\text { abajo: }\end{array}$ & $\begin{array}{l}\text { 2-m identificar } \\
\text { variables }\end{array}$ & $1-\mathrm{a}, 1-\mathrm{c}$ \\
\hline \begin{tabular}{|l} 
11. c. Discuta sus resultados con \\
otros grupos del laboratorio. \\
¿Esos resultados son \\
consistentes con los suyos? \\
Agrupe sus datos y \\
complete la Tabla 2 \\
\end{tabular} & $\begin{array}{l}\text { 1-a Dar razón } \\
\text { 2-o Analizar resultados } \\
\text { 1-d Producción de } \\
\text { razones } \\
\text { 2-j La capacidad de } \\
\text { buscar } \\
\end{array}$ & $\begin{array}{l}\text { 1-a,1-b,1-c,2-a,2-b,2-c,2- } \\
\text { d,3-a,3-b }\end{array}$ \\
\hline $\begin{array}{l}11 . \text { d. ¿Es útil recoger los } \\
\text { resultados de varios grupos } \\
\text { del laboratorio? ¿Cómo este } \\
\text { proceso similar a la } \\
\text { investigación científica? }\end{array}$ & $\begin{array}{l}\text { 1-f Fomentar actitud } \\
\text { crítica } \\
\text { 1-a Dar razón }\end{array}$ & 1-a,1-c,2-a,3-a,4-a \\
\hline $\begin{array}{l}\text { 11. e. Si sus resultados fueron } \\
\text { diferentes de los otros } \\
\text { grupos, Sugiera una posible } \\
\text { razón del por qué sus datos } \\
\text { fueron ser diferentes. }\end{array}$ & $\begin{array}{l}\text { 1-b Busqueda de } \\
\text { explicaciones } \\
\text { 1-f Fomentar actitud } \\
\text { crítica }\end{array}$ & 1-a,1-c,2-b,3-a,4-a \\
\hline
\end{tabular}




\begin{tabular}{|c|c|c|}
\hline $\begin{array}{l}\text { 11. f. Si los resultados no fueron } \\
\text { como usted predijo, sugiera } \\
\text { al menos una posible razón } \\
\text { para explicar las diferentes } \\
\text { tasas de fotosíntesis que } \\
\text { usted observó. Use su } \\
\text { conocimiento acerca de las } \\
\text { plantas y la fotosíntesis para } \\
\text { dar su respuesta. }\end{array}$ & 1-a Dar razón & $1-a, 1-c, 2-a$ \\
\hline $\begin{array}{l}\text { 11. g. Revise la Hipótesis: Basado } \\
\text { en sus datos revise su } \\
\text { hipótesis. }\end{array}$ & $\begin{array}{l}\text { 1-d Producción de } \\
\text { razones }\end{array}$ & $1-b, 3-a$ \\
\hline $\begin{array}{l}\text { 11. h. Describa cómo usted } \\
\text { prepararía el experimento } \\
\text { para probar la hipótesis que } \\
\text { revisó. }\end{array}$ & $\begin{array}{l}\text { 2-e Planear un } \\
\text { experimento sencillo }\end{array}$ & $1-b, 3-a$ \\
\hline $\begin{array}{l}\text { 11. i... ¿Qué otros factores } \\
\text { (además de la tasa de } \\
\text { fotosíntesis) pueden estar } \\
\text { involucrados en la habilidad } \\
\text { de los discos de la hoja para } \\
\text { flotar? }\end{array}$ & $\begin{array}{l}\text { 1-d Producción de } \\
\text { razones } \\
\text { 2-m identificar } \\
\text { variables }\end{array}$ & 1-a,1-b,1-c,3-a \\
\hline $\begin{array}{l}\text { 11. j. Anexo. } \\
\text { Escriba el procedimiento } \\
\text { para el experimento que } \\
\text { usted diseñó }\end{array}$ & $\begin{array}{l}\text { 2-e Planear un } \\
\text { experimento sencillo } \\
\text { 2-n Seleccionar } \\
\text { experimentos } \\
\text { adecuados }\end{array}$ & 1-b,3-a,3-b \\
\hline
\end{tabular}

Tabla 4. Contrastación de Unidades de análisis de la guía de laboratorio e indicadores de las competencias Explicar e Indagar e indicadores para las dimensiones de Comprensión

Así,cuanto a los indicadores de la competencia explicar encontramos que los desempeños que se favorecen en mayor proporción son en su orden (Tabla 5):

\begin{tabular}{|l|c|}
\hline \multicolumn{1}{|c|}{ 1. EXPLICAR } & $\begin{array}{c}\text { NÚMERO DE VECES QUE } \\
\text { SE PRESENTA EN LAS } \\
\text { UNIDADES DE ANÁLISIS }\end{array}$ \\
\hline a. Dar razón de fenómenos o problemas & 5 \\
\hline b. Búsqueda de explicaciones. & 5 \\
\hline d. Producción de razones & 2 \\
\hline f. Fomentar la actitud crítica. & 2 \\
\hline e. Causa y relación con fenómenos & 1 \\
\hline c. Formulación de preguntas. & 0 \\
\hline
\end{tabular}


Tabla 5 Resultados de indicadores para la competencia explicar que más se favorecen en a guía de laboratorio

En cuanto a los indicadores de la competencia Indagar se encontró que los desempeños que se favorecen en mayor proporción son (Tabla 6):

Tabla 6. Resultados de indicadores para la competencia Indagar que más se favorecen en la guía de laboratorio. En cuanto a los indicadores de comprensión se encontró lo siguiente

\begin{tabular}{|l|c|}
\hline \multicolumn{1}{|c|}{ 2. INDAGAR } & $\begin{array}{c}\text { NÚMERO DE VECES QUE } \\
\text { SE PRESENTA EN LAS } \\
\text { UNIDADES DE ANÁLISIS }\end{array}$ \\
\hline $\begin{array}{l}\text { c. } \text { Establecer elementos para comprenderla } \\
\text { resultados. }\end{array}$ & 3 \\
\hline $\begin{array}{l}\text { a. } \text {. obsealizar mediciones y organizar y analizar } \\
\text { d. Búsqueda de información. }\end{array}$ & 2 \\
\hline e. Planear un experimento sencillo & 2 \\
\hline $\begin{array}{l}\text { j. La capacidad de buscar, recoger, } \\
\text { seleccionar información }\end{array}$ & 2 \\
\hline l. Predecir & 2 \\
\hline m. identificar variables & 2 \\
\hline g. buscar relaciones de causa-efecto & 2 \\
\hline h. Hacer predicciones & 1 \\
\hline k. Consulta de libros & 1 \\
\hline n. Seleccionar experimentos adecuados & 1 \\
\hline o. analizar resultados & 1 \\
\hline b. Establecer preguntas pertinentes & 1 \\
\hline f. Planteamiento de nuevas preguntas & 0 \\
\hline
\end{tabular}


Tabla 7. Resultados de indicadores para las dimensiones de comprensión que más se favorecen en la guía de laboratorio

\begin{tabular}{|c|c|}
\hline $\begin{array}{c}\text { DIMENSIONES E INDICADORES DE LA } \\
\text { COMPRENSIÓN }\end{array}$ & $\begin{array}{c}\text { NÚMERO DE VECES } \\
\text { QUE SE PRESENTA } \\
\text { EN LA GUÍA }\end{array}$ \\
\hline \multicolumn{2}{|l|}{ 1. CONOCIMIENTO } \\
\hline a. Manejo de conceptos disciplinarios & 15 \\
\hline c. Dominio del tema & 12 \\
\hline b. Redes altamente organizadas & 7 \\
\hline \multicolumn{2}{|l|}{ 2. MÉTODO } \\
\hline b. Lo hace por el mismo & 9 \\
\hline a. Discusión & 7 \\
\hline c. Preguntas al profesor & 3 \\
\hline d. Preguntas a los compañeros & 2 \\
\hline \multicolumn{2}{|l|}{ 3. PROPÓSITO } \\
\hline a. Reflexión & 11 \\
\hline b. Se desenvuelve fácilmente & 8 \\
\hline \multicolumn{2}{|l|}{$\begin{array}{l}\text { 4. FORMA } \\
\end{array}$} \\
\hline a. Toma en cuenta la opinión de los demás & 2 \\
\hline
\end{tabular}

\section{Contenidos del trabajo práctico}

- Contenido teórico - conceptual del trabajo práctico.

Dentro de la práctica de laboratorio se tienen en cuenta los siguientes fundamentos teóricos: Estructura de la hoja, células del mesófilo y sus características, qué son las tasas de fotosíntesis, cloroplastos y su estructura, reacción lumínica y producción de oxígeno.

- Contenidos procedimentales del trabajo práctico.

Los objetivos básicos de la práctica de laboratorio son medir y comparar las tasas de fotosíntesis de una hoja joven y una hoja adulta.

Las habilidades investigativas que se pretender desarrollar en la guía de laboratorio son:

- Predecir

- Formular hipótesis comprobables

- Analizar datos

- Evaluar y revisar hipótesis. 
Según del Carmen (2000) las habilidades para evaluar las actividades de laboratorio permiten analizar de manera detallada los procedimientos implicados en está práctica de laboratorio, se encontró que se cumplen casi todas excepto aquellas relacionadas con la formulación de preguntas y replanteamiento del problema a investigar.

\section{- Contenidos actitudinales del trabajo práctico.}

Aunque los contenidos actitudinales no son explícitos a la hora de leer la guía de laboratorio, luego de su análisis se pueden deducir las siguientes:

- Asignar y asumir unos roles en la realización de la práctica (un líder y otros que se encarguen de las diferentes actividades, entre otros).

- Cuando se recolecta la información se presupone que se deben respetar los resultados obtenidos y los de los demás

- Durante la argumentación en algunas preguntas se pide un análisis más allá de los que aparece en la misma o de lo que se obtuvo.

- Disposición para el trabajo y para traer los materiales que deben alistar con anticipación.

- Se adquiere una responsabilidad a la hora de entregar el trabajo tanto de prelaboratorio como el informe final.

\section{Caracterización epistemológica}

\begin{tabular}{|c|c|}
\hline LA GUÍA & (CANGUILHEM 1976) \\
\hline \multirow{4}{*}{$\begin{array}{l}\text { La experiencia se realizó con una } \\
\text { parte del organismo. ¿Es posible } \\
\text { medir unas ratas reales conociendo } \\
\text { que solamente es una parte del } \\
\text { individuo que ya no hace parte de } \\
\text { toda la unidad funcional? }\end{array}$} & $\begin{array}{l}\text { Para Canguilhem (1976) Es un hecho } \\
\text { epistemológico que una situación } \\
\text { experimental mostrado de esta manera no } \\
\text { tiene ningún sentido biológico. }\end{array}$ \\
\hline & $\begin{array}{l}\text { Es preciso saber que si se descompone el } \\
\text { organismo viviente aislando sus partes no } \\
\text { es más que para la facilidad del análisis } \\
\text { experimental y no para concebirlas } \\
\text { separadamente. (Canguilhem, 1976) }\end{array}$ \\
\hline & $\begin{array}{l}\text { Ninguna adquisición de carácter } \\
\text { experimental puede ser generalizada sin } \\
\text { expresar reservas, ya sea que se trate de } \\
\text { estructuras, de funciones y de } \\
\text { comportamientos. (Cangilhem, 1976) }\end{array}$ \\
\hline & $\begin{array}{l}\text { Se debe tener una identidad del organismo } \\
\text { de dónde se obtuvo. (Canguilhem, 1976) }\end{array}$ \\
\hline
\end{tabular}

Tabla 8 Caracterización Epistemológica de acuerdo a Canguilhem (1976) 


\section{Caracterización de las actividades de laboratorio.}

Según del Carmen (2000) se analizan ocho dimensiones expresadas en forma de preguntas, tomadas del inventario de dimensiones para evaluar el trabajo práctico (LDI "The Laboratory Dimensions Inventory").

\begin{tabular}{|c|c|c|}
\hline DIMENSIONES & $\begin{array}{c}\text { PREGUNTAS PARA EVALUAR EL } \\
\text { TRABAJO PRÁCTICO }\end{array}$ & $\begin{array}{c}\text { GUÍA DE } \\
\text { LABORATORIO }\end{array}$ \\
\hline \multirow[t]{4}{*}{$\begin{array}{l}\text { 1. Dimensión } \\
\text { social }\end{array}$} & $\begin{array}{l}\text { ¿Los estudiantes trabajan individualmente } \\
\text { o en pequeño grupo? }\end{array}$ & $\begin{array}{l}\text { En pequeños } \\
\text { grupos }\end{array}$ \\
\hline & $\begin{array}{l}\text { ¿Investigan todos la misma cuestión o } \\
\text { aspectos diferentes que después ponen en } \\
\text { común? }\end{array}$ & $\begin{array}{l}\text { Todos la misma } \\
\text { cuestión }\end{array}$ \\
\hline & $\begin{array}{l}\text { ¿Han de discutir los resultados después de } \\
\text { la práctica? }\end{array}$ & Si \\
\hline & $\begin{array}{l}\text { ¿Se establecen relaciones con aplicaciones } \\
\text { sociales? }\end{array}$ & No \\
\hline \multirow[t]{2}{*}{$\begin{array}{l}\text { 2. Conocimientos } \\
\text { previos }\end{array}$} & $\begin{array}{l}\text { ¿Qué conocimientos se necesitan para } \\
\text { poder realizar adecuadamente el trabajo } \\
\text { práctico? }\end{array}$ & $\begin{array}{l}\text { Estructura de la } \\
\text { hoja. } \\
\text { Célula vegetal } \\
\text { Cloroplasto } \\
\text { Reacciones } \\
\text { lumínicas } \\
\text { Relación entre luz } \\
\text { y pigmentos }\end{array}$ \\
\hline & $\begin{array}{l}\text { ¿Poseen las habilidades técnicas necesarias } \\
\text { para su realización? }\end{array}$ & Si \\
\hline \multirow[t]{3}{*}{$\begin{array}{l}\text { 3. Relación con la } \\
\text { teoría }\end{array}$} & $\begin{array}{l}\text { ¿Se considera que la teoría es básica para } \\
\text { realizar la investigación? }\end{array}$ & $\mathrm{Si}$ \\
\hline & $\begin{array}{l}\text { ¿Es necesario encontrar una explicación } \\
\text { teórica a las hipótesis? }\end{array}$ & $\mathrm{Si}$ \\
\hline & $\begin{array}{l}\text { ¿Se pide a los alumnos que relacionen las } \\
\text { conclusiones con la teoría? }\end{array}$ & Todo el tiempo \\
\hline $\begin{array}{l}\text { 4. Obtención de } \\
\text { datos }\end{array}$ & $\begin{array}{lrr}\text { ¿Cómo se obtienen } & \text { los datos?; } \\
\text { observaciones } & \begin{array}{l}\text { directas, } \\
\text { aparatos, computadora. }\end{array} & \text { indicadores, } \\
\end{array}$ & $\begin{array}{l}\text { Observaciones } \\
\text { directas }\end{array}$ \\
\hline $\begin{array}{l}\text { 5. Complejidad de } \\
\text { los instrumentos }\end{array}$ & $\begin{array}{l}\text { ¿La complejidad de los instrumentos es } \\
\text { adecuada a la finalidad que se persigue? }\end{array}$ & $\mathrm{Si}$ \\
\hline $\begin{array}{l}\text { 6. Análisis de } \\
\text { datos }\end{array}$ & $\begin{array}{l}\text { ¿Qué tipo de análisis se pide? } \\
\text { ¿Se orienta a los alumnos sobre la forma } \\
\text { más idónea de expresar, presentar y } \\
\text { comunicar los datos? }\end{array}$ & $\begin{array}{l}\text { Análisis teórico de } \\
\text { contrastación con } \\
\text { la teoría y con } \\
\text { otros grupos. }\end{array}$ \\
\hline
\end{tabular}




\begin{tabular}{|l|l|l|}
\hline 7. Tiempo & $\begin{array}{l}\text { ¿El tiempo necesario para realizar el trabajo } \\
\text { práctico justifica su realización? }\end{array}$ & $\mathrm{Si}$ \\
\hline & $\begin{array}{l}\text { ¿Es compatible con la distribución del } \\
\text { horario de clases? }\end{array}$ & $\mathrm{Si}$ \\
\hline $\begin{array}{l}\text { 8. Aprendizaje de } \\
\text { conceptos }\end{array}$ & $\begin{array}{l}\text { ¿El trabajo práctico está pensado para } \\
\text { enseñar un concepto importante? }\end{array}$ & Sí. \\
\hline & $\begin{array}{l}\text { ¿Ayuda a superar las ideas previas de los } \\
\text { alumnos y aproximarlas a los conceptos } \\
\text { científicos trabajados? }\end{array}$ \\
\hline
\end{tabular}

Tabla 9. Caracterización de la guía de laboratorio (Del Carmen, 2000)

Según Caamaño (2003) la clasificación de esta práctica de acuerdo al objetivo perseguido es:

\begin{tabular}{|l|l|}
\hline \multicolumn{1}{|c|}{$\begin{array}{c}\text { TIPO DE TRABAJO } \\
\text { PRÁCTICO }\end{array}$} & \multicolumn{1}{c|}{ "LABORATORIO PHOTO-FINISH" } \\
\hline Experimento Ilustrativo: & $\begin{array}{l}\text { Ilustra un principio o una relación entre variables. } \\
\text { (Caamaño, 2003) Y es una aproximación cualitativa o } \\
\text { semicualitativa al fenómeno. }\end{array}$ \\
\hline Ejercicio Práctico & $\begin{array}{l}\text { Para Ilustrar la Teoría (Caamaño, 2003) } \\
\text { Se pone énfasis en la determinación experimental de } \\
\text { propiedades y en la comprobación de leyes o relaciones } \\
\text { entre variables, con objetivos ilustrativo o corroborativo } \\
\text { de la teoría y con enfoque dirigido. }\end{array}$ \\
\hline
\end{tabular}

Tabla 10. Clasificación de la práctica de laboratorio según Caamaño (2003)

\section{Valoración inicial de la guía}

Valoración inicial en cuanto a:

\section{Debilidades:}

- Inicialmente la guía no facilitaba la formulación de preguntas

- Tomando en cuenta a lo expuesto por Canguilheim (1976), la experiencia no toma en consideración al organismo completo, ni las condiciones en que se encontraba.

- No se promueven aplicaciones sociales, ni relaciones con su cotidianidad.

\section{Fortalezas:}

En la secuencia de actividades se posibilita

- Introducción por parte del profesor para presentar la cuestión o problema que centrará el trabajo práctico y ubicarlo teóricamente.

- Explicación de la práctica propiamente dicha y trabajo de los alumnos con el grupo 
- Comprobación por parte del profesor de el apropiamiento de algunos conceptos necesarios para la comprensión de lo que se hace en la práctica.

- Una o más actividades de laboratorio y/o campo realizadas por los alumnos

- Elaboración de un resumen y conclusiones individuales y en pequeño grupo

- Comunicación en el grupo clase

- Actividades de sistematización y síntesis

- Actividades de evaluación

- En algunos de sus apartados la guía permite la reflexión acerca de cómo trabajan los científicos e invita a los estudiantes a que compare el trabajo en el ambiente escolar y en el medio científico.

- Promueve la metacognición que posibilita la comprensión

\section{Limitantes:}

- El tiempo que requiere el desarrollo de la guía completa.

Otras observaciones iniciales fueron las siguientes:

Respecto al análisis de los indicadores de las competencias indagar y explicar encontramos que solamente tres de ellas no se posibilitan en la guía estas son:

1-C Formulación de preguntas.

2-B Establecer preguntas pertinentes.

2-F Planteamiento de nuevas preguntas

Por tal motivo, se complementó la guía de laboratorio con tres nuevas preguntas que se agregaron pretendiendo dar alcance a todos los indicadores expuestos.

Las preguntas y su ubicación en la guía fueron las siguientes:

7-c. Elabore dos preguntas que guíen un procedimiento experimental para medir y analizar esos procesos cambiantes.

11-f ¿Qué preguntas le suscitan estos resultados discrepantes?

11-i Escriba la pregunta que se plantearía inicialmente para realizar el experimento.

11-j Elabore una pregunta que permita relacionar alguno de los factores con una evidencia de que el proceso de fotosíntesis está ocurriendo.

\section{Fases finales de Ejecución}

\section{Aplicación de la guía de laboratorio modificada}

Se llevó a cabo la práctica de laboratorio con los estudiantes de los grados 9A, 9B y 9C con un total de 83 estudiantes, para lo cual se contó con su filmación, registro de desempeños 
observados. Luego se realizó la sistematización de la información, se describió, tabuló, organizó en cuadros y se trianguló para su interpretación y análisis.

\section{Filmación}

Se hizo la grabación durante el desarrollo del trabajo práctico en el laboratorio, durante el desarrollo de la práctica se pasaba registrando por cada grupo, se obtuvieron algunas conversaciones entre los estudiantes, a lo que le prestaban atención, distribución de roles para la preparación del material, toma de tiempo, etc y en general fue muy valioso por las preguntas que suscitó el trabajo, la observación constante al proceso y la expectativa por saber cuál de las dos hojas iba a ganar la carrera. Para poder recoger la mayor cantidad posible de información se utilizaron las mismos indicadores de las competencias y de la comprensión, cuando el observador notaba que se presentaba alguno de ellos lo registraba en el formato .
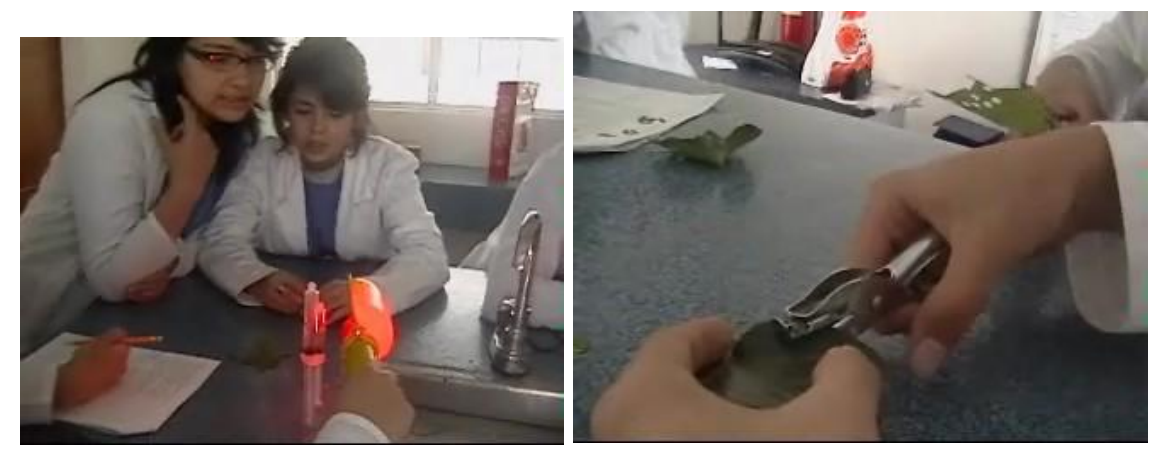

Figura 2. Registros fotográficos durantes el desarrollo de la práctica.

\section{Recolección de Información y análisis}

- Durante la práctica de laboratorio

En el análisis del registro para los indicadores de la Competencia explicar e indagar los que más se favorecieron fueron: la capacidad de buscar, recoger, seleccionar información, analizar resultados realizar mediciones y organizar.

- Después de la práctica de laboratorio 


\begin{tabular}{|l|l|}
\hline \multicolumn{1}{|c|}{ PREGUNTA 7-a } & $\begin{array}{l}\text { Si usted pudiera diseñar una hoja que fotosintetizara } \\
\text { "velozmente" iqué características tendría? (Piense acerca } \\
\text { de la estructura de una hoja y la célula de la planta.) } \\
\text { Explique su razonamiento }\end{array}$ \\
\hline \multicolumn{2}{|c|}{ INDICADORES } \\
\hline \multicolumn{1}{|c|}{ Explicar } & 1-b Búsqueda de explicaciones \\
\hline \multicolumn{1}{|c|}{ RESPUESTA } & 2-1 Predecir \\
\hline Estomas & \multicolumn{1}{c|}{ NUEMO DE REPORTES } \\
\hline Cloroplastos & 7 \\
\hline Células mesofilo & 5 \\
\hline Tilacoides & 2 \\
\hline Clorofila & 1 \\
\hline Espacio para el aire & 1 \\
\hline
\end{tabular}

Para el análisis de los informes de la práctica de laboratorio se tuvo en cuenta cada unidad que los estudiantes tenían que contestar a continuación se muestra una de las preguntas.

Tabla 11. Ejemplo de análisis a las unidades encontradas en la guía de laboratorio.

Para los indicadores de la Competencia explicar e indagar se pudieron extraer los siguientes resultados de los reportes de laboratorio

\begin{tabular}{|l|c|}
\hline \multicolumn{1}{|c|}{ INDICADORES } & $\begin{array}{c}\text { TOTAL REPORTES } \\
\text { DE LABORATORIO }\end{array}$ \\
\hline \multicolumn{1}{|c|}{ 1. EXICAR } & 15 \\
\hline a. Dar razón de fenómenos o problemas & 15 \\
\hline b. Búsqueda de explicaciones. & 8 \\
\hline c. formulación de preguntas. & 12 \\
\hline d. producción de razones & 15 \\
\hline e. causa y relación con fenómenos & 15 \\
\hline f. Fomentar la actitud crítica. & \\
\hline & 15 \\
\hline a. observar detenidamente la situación & 8 \\
\hline b. Establecer preguntas pertinentes & 15 \\
\hline c. Establecer elementos para comprenderla & 15 \\
\hline d. Búsqueda de información. & 11 \\
\hline e. Planear un experimento sencillo & 8 \\
\hline f. Planteamiento de nuevas preguntas & 15 \\
\hline g. buscar relaciones de causa-efecto & 15 \\
\hline h. Hacer predicciones & \\
\hline
\end{tabular}




\begin{tabular}{|l|c|}
$\begin{array}{l}\text { i. } \text { Realizar mediciones y organizar y analizar } \\
\text { resultados. }\end{array}$ & 15 \\
\hline $\begin{array}{l}\text { j. La capacidad de buscar, recoger, seleccionar } \\
\text { información }\end{array}$ & 10 \\
\hline k. Consulta de libros & 15 \\
\hline l. Predecir & 15 \\
\hline m. Identificar variables & 14 \\
\hline n. Seleccionar experimentos adecuados & 11 \\
\hline o. Analizar resultados & 10 \\
\hline
\end{tabular}

Tabla 12. Indicadores de la competencia Explicar e indagar en los reportes de laboratorio

Como se puede observar los indicadores que menos se favorecieron con el reporte de laboratorio fueron aquellos relacionados con la formulación de preguntas, seguido por la capacidad de recolectar información, análisis de resultados, seleccionar experimentos adecuados y planear experimentos sencillos.

Aunque la guía de laboratorio fue modificada muy poco reportes plantearon el procedimiento de un experimento sencillo que se adecuara a la hipótesis de igual manera la de formulación de preguntas no fue desarrollada en su totalidad por todos los grupos.

Para las dimensiones de la comprensión se obtuvieron los siguientes resultados en los reportes de laboratorio:

\begin{tabular}{|c|c|}
\hline INDICADORES & $\begin{array}{l}\text { TOTAL REPORTES } \\
\text { DE LABORATORIO }\end{array}$ \\
\hline \multicolumn{2}{|l|}{ 1. CONOCIMIENTO } \\
\hline a. Manejo de conceptos disciplinarios & 15 \\
\hline b. Redes altamente organizadas & 10 \\
\hline c. Dominio del tema & 8 \\
\hline \multicolumn{2}{|l|}{ 2. MÉTODO } \\
\hline a. Discusión & 15 \\
\hline b. Lo hace por el mismo & 15 \\
\hline \multicolumn{2}{|l|}{ c. Preguntas al profesor } \\
\hline \multicolumn{2}{|l|}{ d. Preguntas a los compañeros } \\
\hline \multicolumn{2}{|l|}{ 3. PROPÓSITO } \\
\hline a. Reflexión & 15 \\
\hline b. Se desenvuelve fácilmente & 8 \\
\hline \multicolumn{2}{|l|}{ 4. FORMA } \\
\hline a. Toma en cuenta la opinión de los demás & \\
\hline
\end{tabular}

Tabla 13. Indicadores de la comprensión en los reportes de laboratorio 
Los indicadores que menos se favorecieron con el reporte de laboratorio fueron aquellos relacionados con el dominio del tema, el desenvolverse fácilmente y redes altamente organizadas, algunos estudiantes pueden conocer los conceptos pero no comprender sus relaciones y aplicaciones biológicas.

En cuanto al manejo conceptual y comprensión del proceso algunos estudiantes pueden conocer los conceptos pero no comprender sus relaciones y aplicaciones biológicas. Algunas explicaciones se remontan a diferentes factores entre los que sobresalen los estructurales debido a la cantidad de estomas o cloroplastos; para plantear este tipo de explicaciones es necesario que el estudiante haya adquirido con anticipación un bagaje conceptual que le permita comprender el proceso e identificar los factores involucrados.

\section{CONCLUSIONES}

El presente trabajo de investigación permite concluir que si se tienen las condiciones de tiempo, de formación y de disposición los profesores podemos hacer investigación acción para el mejoramiento de nuestra práctica pedagógica a favor de los aprendizajes de los estudiantes. Para ello es importante en cuanto a las prácticas de laboratorio, tener claros los objetivos que se pretender alcanzar y estar fundamentado metodológicamente.

La investigación realizada permitió evaluar el desarrollo de las competencias científicas explicar e indagar en el marco de la enseñanza para la comprensión mediante la aplicación de un trabajo práctico sobre fotosíntesis en tres grupos de estudiantes de noveno grado, a través del seguimiento de una serie de indicadores del desempeño de los estudiantes lo que permitió reformar la guía de laboratorio y mejorarla para dar alcance a todos ellos.

Se halla coherencia entre las habilidades que se pretendía desarrollar con la guía de laboratorio y las que realmente se evidenciaron a través de las dimensiones de la comprensión y los desempeños de las competencias científicas como predecir, formular, evaluar y revisar hipótesis, y analizar datos. Algunos de los elementos del modelo de la enseñanza para la comprensión sobresalientes durante la evaluación del trabajo práctico de laboratorio sobre fotosíntesis, respecto de contenido, método, propósito y forma fueron manejo de conceptos, formulación de preguntas y reflexión y finalmente la reestructuración de la guía permitió complementarla con algunos indicadores como formulación de preguntas y constante reflexión, demostrando que es importante evaluar los desempeños de comprensión y habilidades investigativas.

La definición de las categorías de análisis a partir de los distintos ítems de la guía y el contrastarlos con los desempeños en torno a las competencias, se constituyó en una excelente metodología para verificar el desarrollo tanto de los distintos indicadores de estas competencias, como de las dimensiones de la comprensión.

Se puede afirmar que el planteamiento didáctico de la misma práctica pedagógica de un docente se constituye en un referente de análisis, que permite valorar cada una de las 
etapas identificadas en su hacer, frente a los cuales es también posible establecer su impacto respecto a los logros de los estudiantes.

Apoyados en los distintos referentes del marco teórico revisado fue posible estructurar criterios orientadores para la valoración cualitativa de los elementos planteados en la guía de laboratorio de fotosíntesis. Al aplicar los criterios definidos para la valoración de la guía inicialmente propuesta fue posible identificar las debilidades y fortalezas de la guía propuesta y orientar su complementación para su mejor aprovechamiento en cuanto al aprendizaje de los estudiantes. Estos criterios permitieron hacer una evaluación coherente de las competencias, logrando que los estudiantes asumieran una posición reflexiva en cuanto a la formulación de preguntas.

Las relaciones que se establecen en los contenidos teóricos de la guía exigen que el estudiante se haya apropiado con anticipación de unos conceptos fundamentales en cuanto al proceso lumínico que se lleva a cabo en las plantas y de la misma estructura de la célula vegetal. En cuanto a los contenidos procedimentales el desarrollo no requiere de habilidades técnicas elevadas pero sí de desempeños importantes de indagación y explicación útiles en diferentes áreas del conocimiento por ende aplicable a diferentes contextos.

En el laboratorio se manejan ciertas condiciones que varían de las encontradas en el medio natural, estas variables controladas requieren ciertos niveles de comprensión soportados tanto por los procedimientos, como por lo teórico. Algunos estudiantes en este caso alcanzan en cuanto al modelo de enseñanza para la comprensión un nivel de maestría al reflexionar en cuanto a las unidades biológicas que están manipulando en el desarrollo del trabajo práctico.

Este tipo de investigaciones demuestran que el profesor tiene a su disposición materiales educativos que le brindan posibilidades para lograr de ellos un mejor aprovechamiento y en él mismo proceso una cualificación, permitiendo hacer una reflexión constante y evaluar constantemente los resultados obtenidos en diferentes momentos de la práctica pedagógica. De igual manera es importante determinar los desempeños y habilidades que el estudiante desarrolla durante el proceso de aprendizaje. El estudiar lo que sucede en el aula de ciencias naturales permite hacer diferentes interpretaciones que orientan su transformación y aprender desde la misma reflexión.

\section{BIBLIOGRAFÍA}

BLYTHE, T. 1999. La enseñanza para la comprensión. Guía para el docente. Ed. Paidos. Buenos Aires. Argentina.

CAAMAÑO, A. 2003. Los trabajos practicas en ciencias. En: Jiménez, P. (Compiladora). Enseñar Ciencias. Barcelona: Graó, pp. 95. 
CAMPBELL, N., WILLIAMSON, B.,HEYDEN, R. 2004. Biology. Exploring Life. Ed. Prentice Hall, p. 848.

CALGUILHEM, G. 1976. La experimentación en Biología Animal. El Conocimiento de la Vida. Ed. Anagrama. Barcelona España.

CERDA, H. 2005. Los elementos de la investigación. Como diseñarlos y construirlos. Bogotá. Col. Ed. El Buho.

CHARRIER, M, CAÑAL, P., RODRIGO, M. 2006. Las concepciones de los estudiantes sobre la fotosíntesis y la respiración: una revisión sobre la investigación didáctica en el campo de la enseñanza y el aprendizaje de la nutrición de las plantas una revisión sobre la investigación didáctica en el campo de la enseñanza y el aprendizaje de la nutrición de las plantas. Enseñanza de las ciencias, 24(3).

DEL CARMEN, L. 2000. Los trabajos prácticos. En: Perales, F., Cañal, P (compiladores). Didáctica de las Ciencias Experimentales. Teoría y práctica de la Enseñanza de la Ciencias. Alcoy: Marfil, p.p. 267-288.

FEDERMAN, J., QUINTERO, J., ANCIZAR, R. Competencias Investigativas para profesionales que forman y enseñan. ¿Cómo desarrollarlas?. Aula Abierta Magisterio. P. 253 Bogotá Colombia.

INSTITUTO COLOMBIANO PARA LA EDUCACIÓN SUPERIOR. ICFES. 2001. Fundamentación Conceptual área de Ciencias Naturales. Bogotá. 2007.

STONE, M. 1998. La enseñanza para la comprensión. Vinculación entre la Investigación y la práctica. Ed. Paidos. Buenos Aire. Argentina. 


\section{Guía de Laboratorio.}

Laboratorio $\mathrm{N}^{\circ} 8$

Photo Finish

\section{Comparando rata fotosintética.}

1. Pregunta. ¿Cuál hoja fotosintetizará a una rata más rápida, una joven o una adulta?

2. Apreciación Global. En esta investigación, usted comparará tasas de fotosíntesis en una hoja joven y en una hoja adulta midiendo el tiempo

que toman los discos de cada tipo de hoja para generar suficiente

gas y flotar en una jeringa llena de una solución.

3. Introducción. Recordar que los cloroplastos se localizan en las células del mesófilo, el tejido verde en el interior de una hoja. Las células del mesófilo tienen los espacios de aire alrededor de ellas para la salida de gases. Usted debe tener en cuenta estas funciones y las características estructurales de la hoja para medir y comparar la rata fotosintética en ellas. La rata indica cómo cambia la cantidad medible cuando pasa el tiempo. Como el oxígeno es un gas producto de fotosíntesis, la rata de fotosíntesis en una hoja puede determinarse midiendo la cantidad de oxígeno que una hoja produce en un cierto período de tiempo. En esta investigación, usted medirá la producción de oxígeno indirectamente. Suspenderá los discos de la hoja en una solución y aplicará un vacío. Se llenarán los espacios de aire dentro de cada disco de la hoja con el líquido y los discos de la hoja se hundirán. Usted contará entonces los discos de la hoja que flotan. Cuando las reacciones lumínicas producen oxígeno, el líquido es forzado fuera de los espacios de aire y los discos de la hoja flotarán En general, cuanto más rápido floten los discos de la hoja más rápido estarán fotosintetizando.

4. Actividad de Prelaboratorio. Compare las hojas competidoras mostrada y lea la información que aparece abajo. Luego conteste las preguntas

Distancia final: $10 \mathrm{cc}$

\section{Competidor 1 \\ Hoja verde, joven, activamente en \\ Crecimiento}

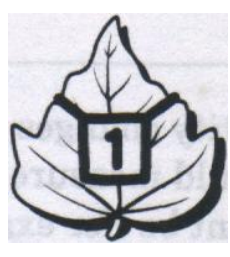

\section{Competidor 2}

Hoja verde

oscura,

adulta, crecimiento completo.

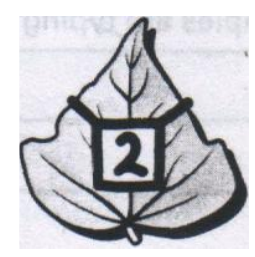


1. Pregunta. ¿Cuál hoja fotosintetizará a una rata más rápida, una joven o una adulta?

2. Apreciación Global. En esta investigación, usted comparará tasas de fotosíntesis en una hoja joven y en una hoja adulta midiendo el tiempo que toman los discos de cada tipo de hoja para generar suficiente gas y flotar en una jeringa llena de una solución.

3. Introducción. Recordar que los cloroplastos se localizan en las células del mesófilo, el tejido verde en el interior de una hoja. Las células del mesófilo tienen los espacios de aire alrededor de ellas para la salida de gases. Usted debe tener en cuenta estas funciones y las características estructurales de la hoja para medir y comparar la rata fotosintética en ellas. La rata indica cómo cambia la cantidad medible cuando pasa el tiempo. Como el oxígeno es un gas producto de fotosíntesis, la rata de fotosíntesis en una hoja puede determinarse midiendo la cantidad de oxígeno que una hoja produce en un cierto período de tiempo. En esta investigación, usted medirá la producción de oxígeno indirectamente. Suspenderá los discos de la hoja en una solución y aplicará un vacío. Se llenarán los espacios de aire dentro de cada disco de la hoja con el líquido y los discos de la hoja se hundirán. Usted contará entonces los discos de la hoja que flotan. Cuando las reacciones lumínicas producen oxígeno, el líquido es forzado fuera de los espacios de aire y los discos de la hoja flotarán En general, cuanto más rápido floten los discos de la hoja más rápido estarán fotosintetizando.

\section{Una carrera de fotosíntesis}

La carrera tendrá lugar en dos jeringas llenas de solución. Usted suspenderá 10 discos de las hojas en cada jeringa, empujará el émbolo hacia atrás para aplicar vacío, y permitirá que el aire dentro de las hojas fluya hacia afuera. Cuando el aire sea reemplazado por el líquido, los discos de la hoja se hundirán y esta será la línea de partida que es el fondo de la jeringa. Cuando usted ponga ambas jeringas cerca de la fuente lumínica para empezar la fotosíntesis, la carrera empezará. Cuando los discos de la hoja producen el oxígeno empiezan a flotar, ellos correrán hasta la meta que es la cima de la jeringa.

\section{Ganando la carrera de la fotosíntesis}

Cuando 5 de los 10 discos de una misma hoja hayan alcanzado la cima de la jeringa, el "corredor" de la hoja habrá cruzado la "línea de llegada", y usted registrará el tiempo.

\section{Preguntas Previas}

a. Si usted pudiera diseñar una hoja que fotosintetizara "velozmente", qué características tendría? (Piense acerca de la estructura de una hoja y la célula de la planta.) Explique su razonamiento.

b. Qué hoja de hiedra fotosintetizará más rápido y ganará la carrera? Explique el por qué de su predicción. (Ésta es su hipótesis.) 
c. Una tasa mide cómo una cantidad cambia con el tiempo. Dé un ejemplo de algo a lo que usted podría medirle una rata de cambio. ¿Qué unidades de medida serían (por ejemplo, metros/segundos)?

\section{Materiales}

- Dos hojas de Hiedra: una oscura y adulta la otra verde claro y joven.

- Dos jeringas

- Marcador

- Abre huecos

- Bicarbonato/solución detergente en un vaso plástico.

- Fuente de luz fuerte

- Cronometro.

\section{Procedimiento}

1. Obtener dos hojas de hiedra una joven y verde (Corredor 1) y otra adulta y oscura (Corredor 2).

2. Corte 10 discos de cada hoja con el abre huecos.

3. Coloque los discos de las hojas dentro del barril de la jeringa inmediatamente márquela.

4. Repita pasos 1-3 para la hoja oscura (Corredor 2)

5. Después de que las dos jeringas estén listas, Empuje los émbolos hasta que estas toquen los discos de las hojas. Tenga cuidado de no presionarlas demasiado.

6. Prepare aproximadamente 5 centímetros cúbicos de bicarbonato de sodio y solución detergente dentro de cada jeringa.

7. Invierta la jeringa (colóquela al revés) y agítela para que produzca las burbujas.

8. Empuje hacia adentro los émbolos para mover todo el aire fuera de los extremos de las jeringas.

9. Coloque su dedo encima de la punta de una jeringa mientras tira el embolo hacia atrás hasta 10 cc mostrado abajo. No lo hale demasiado ya que podría sacarlo esto crea un vacío, mientras tanto el aire se mueve

10. Sostenga el vacío manteniendo el dedo en la punta de la jeringa y agite varias veces para

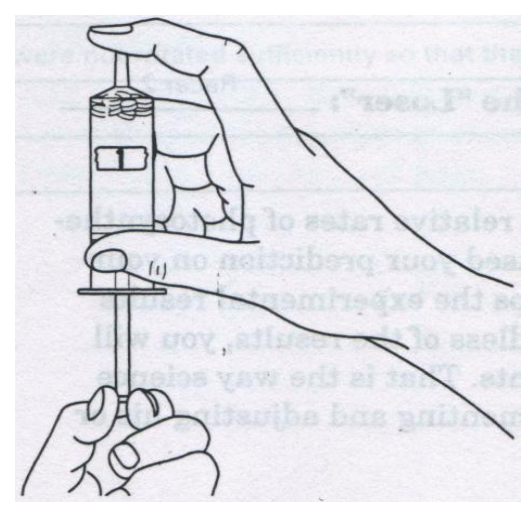


producir las burbujas.

11. Con la punta de la jeringa apuntando hacia arriba retire su dedo y observe si las hojas se hunden. Si ellas lo hacen coloque la jeringa en frente de la fuente de luz (La cual debe estar apagada). Si alguna hoja se mantiene flotando repita pasos 8-11 hasta que se hunda. Esto puede tomar varias pruebas. Si hay un o dos discos tercos, simplemente déjeles flotando.

12. Después de que ambos corredores están en punto de arranque "cerca de la fuente de luz”, encienda la luz y de la salida cronometrando. ¡La carrera empieza! Cuándo vea un disco subir a la cima, registre el tiempo y total de discos que han subido en la tabla de datos 1 .

a. Tiempo de partida de la carrera:

\section{b. Tabla de Datos 1}

\begin{tabular}{|l|c|c|}
\hline $\begin{array}{c}\text { Tiempo en } \\
\text { Minutos y } \\
\text { Segundos }\end{array}$ & $\begin{array}{c}\text { Corredor } \mathbf{1} \\
\text { (Hoja Verde } \\
\text { Clara) }\end{array}$ & $\begin{array}{c}\text { Corredor 2 } \\
\text { (Hoja Verde } \\
\text { Oscura) }\end{array}$ \\
\hline $\begin{array}{l}\text { Tiempo Partida: } 0 \\
\text { minutos }\end{array}$ & 0 discos arriba & 0 discos arriba \\
\hline & & \\
\hline & & \\
\hline & & \\
\hline
\end{tabular}

13. Cada 2 o 3 minutos usted puede girar ambas jeringas para recuperar cualquier disco que se pegue a las paredes de la jeringa. Cuando 5 de los 10 discos estén flotando en la cima, ha terminado la carrera. Siga tomando el tiempo y registrando los datos hasta que ambos corredores hayan terminado.

\section{Resultados de la Carrera:}

\section{El "Ganador":}

El "perdedor":

\section{Análisis y Conclusiones}

Antes de que la carrera empezara, usted predijo las ratas relativas de fotosíntesis -de las dos hojas diferentes de hiedra. Usted basó su predicción entendiendo la fotosíntesis. Quizás los resultados experimentales no salgan como usted esperó. Sin tener en cuenta los resultados, usted considerará la necesidad de realizar más experimentos. Ésa es la manera como los científicos trabajan. Cada científico debe seguir experimentando y ajustando sus hipótesis basándose en los datos. 
a. Predijo usted al ganador?

b. Calcule la rata de fotosíntesis del ganador completando el cálculo abajo:

5 discos arriba / minutos $=$

discos/min:

c. Discuta sus resultados con otros grupos del

laboratorio. ¿Esos resultados son consistentes con los suyos? Agrupe sus datos y complete la Tabla 2.

Tabla de Datos 2:

\begin{tabular}{|l|l|l|}
\hline $\begin{array}{c}\text { Grupo de } \\
\text { laboratorio }\end{array}$ & $\begin{array}{c}\text { Hoja más } \\
\text { rápida }\end{array}$ & Hoja más lenta \\
\hline & & \\
\hline & & \\
\hline & & \\
\hline & & \\
\hline & & \\
\hline
\end{tabular}

Resultados Esperados

En general los discos de la Hoja joven van a fotosinte tizar más rápido que los de la hoja adulta.

\section{Resultados}

d. Es útil recoger los resultados de varios grupos del laboratorio? ¿Cómo se asemeja este proceso a la investigación científica?

e. Si sus resultados fueron diferentes de los otros grupos, Sugiera una posible razón del por qué sus datos fueron ser diferentes.

f. Si los resultados no fueron como usted predijo, sugiera al menos una posible razón para explicar las diferentes ratas de fotosíntesis que usted observó. Use su conocimiento acerca de las 
Bio-grafia: Escritos sobre la Biología y su Enseñanza Vol 3 No4 ISSN 2027-1034. Primer semestre de 2010, Bogotá, Colombia, pp 28-57

plantas y la fotosíntesis para dar su respuesta. -

g. Revise la Hipótesis: Basado en sus datos revise su hipótesis.

h. Describa cómo prepararía usted un experimento para probar la hipótesis revisada.

i. Qué otros factores (además de la rata de fotosíntesis) pueden estar involucrados en la habilidad de los discos de la hoja para flotar?

j. Anexo.

Escriba el procedimiento para el experimento que usted diseñó en la pregunta 8 para probar la hipótesis que revisó. Tenga en cuenta la aprobación del profesor. Después, con el permiso de su maestro, lleve a cabo el experimento. $Y$ finalmente, comparta sus datos y conclusiones con la clase. 
Bí-grafia: Escritos sobre la Biología y su Enseñanza Vol 3 No4 ISSN 2027-1034. Primer semestre de 2010, Bogotá, Colombia, pp 28-57 